This item was submitted to Loughborough's Research Repository by the author.

Items in Figshare are protected by copyright, with all rights reserved, unless otherwise indicated.

\title{
A uniqueness criterion for linear problems of wave-body interaction
}

PLEASE CITE THE PUBLISHED VERSION

LICENCE

CC BY-NC-ND 4.0

\section{REPOSITORY RECORD}

Motygin, O.V., and P. Mclver. 2019. "A Uniqueness Criterion for Linear Problems of Wave-body Interaction". figshare. https://hdl.handle.net/2134/455. 


\title{
A uniqueness criterion for linear problems of wave-body interaction
}

\author{
O.V. Motygin ${ }^{1}$ AND P. MCIVER ${ }^{2}$ \\ ${ }^{1}$ Institute of Problems in Mech. Engineering, Russian Academy of Sciences, \\ V.O., Bol'shoy pr. 61, 199178 St Petersburg, Russian Federation \\ E-mail: mov@snark.ipme.ru \\ ${ }^{2}$ Department of Mathematical Sciences, Loughborough University, \\ Loughborough, Leics., LE11 3TU, United Kingdom \\ E-mail: p.mciver@lboro.ac.uk
}

\begin{abstract}
The question of uniqueness for linearized problems describing interaction of submerged bodies with an ideal unbounded fluid is far from its final resolution. In the present work a new criterion of uniqueness is suggested based on Green's integral identity and maximum principles for elliptic differential equations. The criterion is formulated as an inequality involving integrals of the Green function over the bodies' wetted contours. This criterion is quite general and applicable for any number of submerged bodies of fairly arbitrary shape (satisfying an exterior sphere condition) and in any dimension; it can also be generalised to more complicated elliptic problems. Very simple bounds are also derived from the criterion, which deliver uniqueness sets in the space of parameters defined by submergence of the system of bodies and the frequency of oscillation. Results of numerical investigation and comparison with known uniqueness criteria are presented.
\end{abstract}

\section{Introduction}

This article is concerned with the two and three-dimensional linear boundaryvalue problems of the interaction between an ideal unbounded fluid and bodies located under the free surface of the fluid. Two classes of problem, describing the radiation of waves by the forced motion of rigid bodies and the diffraction of waves by fixed rigid bodies, appear within the framework of the surface wave theory under the usual assumptions that the motion is steady-state, irrotational and the oscillations have small amplitudes.

Our interest here is in the question of uniqueness. For these seemingly simple problems very few criteria of uniqueness are known despite the long history of 
surface-wave theory and the importance of the uniqueness property (see Ursell (1992), where this question is placed first in a list of unfinished problems).

In order to explain the difficulty in investigating the uniqueness question we note that uniqueness in the problems under consideration is known to be equivalent to non-existence of so-called trapped modes, that is non-trivial localised solutions of the homogeneous boundary-value problem. At the same time, the problems in the absence of bodies allow wave solutions propagating to infinity, and hence trapped modes are found within the space of functions having infinite energy and the frequencies of trapped modes can be considered as point eigenvalues embedded in the continuous spectrum. This case is much more difficult to analyse than that of non-embedded eigenvalues.

An extensive review of existing results on uniqueness can be found, e.g. in Kuznetsov et al. (2002, part I), but two main results should be mentioned here. In Vainberg \& Maz'ya (1973) the so-called Maz'ya's identity was introduced (see also Maz'ya, 1978; Hulme, 1984), which allows uniqueness to be proven at all frequencies for particular body geometries such that some special vector fields no-where enter the wetted surface of the bodies. Another scheme was used for the two-dimensional problem by Simon \& Ursell (1984) who extended the approach of John (1950) to prove uniqueness for any system of submerged bodies which are contained between lines which intersect the free surface at the same point and at angle $45^{\circ}$ to the horizontal; for lines that are not straight the possible inclinations are greater. The technique used by Simon \& Ursell (1984) also shows that the potential will be unique provided that the frequency is small or large enough. In either limit, the method gives a bound on how small, or large, the frequency must be for uniqueness to be guaranteed. An improvement of the result by Simon \& Ursell (1984) for the case when the submerged structure is symmetric about a vertical line (two-dimensional problem) or axisymmetric (three-dimensional problem) can be found in Kuznetsov \& Simon (1999).

One might expect that it would be possible to improve the results of Vainberg \& Maz'ya (1973) or Simon \& Ursell (1984) to include much wider, if not all, classes of geometries, at least for the case of totally submerged bodies. However, in recent years a number of examples of non-uniqueness (that is structures that support trapped modes) have been constructed. This was first done for particular surface-piercing bodies by McIver (1996) and for totally submerged bodies by McIver (2000) by using the so-called inverse scheme, in which the geometry of a trapping configuration is sought for a given potential. Other examples of non-uniqueness for totally submerged bodies are constructed in McIver \& Porter (2002) and Porter (2002), where numerical techniques are developed for finding trapped modes for given configurations and results are presented for submerged elliptical toroids and for a variety of symmetrical pairs of submerged obstacles. Thus, it is now clear that no general uniqueness proof is possible and, in particular, the angle in the proof by Simon \& Ursell (1984) can not be decreased to zero; structures found in Porter (2002) have a maximum angle of about $6.7^{\circ}$. 
In the present work we shall present a new criterion for uniqueness based on use of the Green identity and maximum principles. The basic approach has a quite general nature and can be applied to elliptic problems with more complicated operators than that in the present paper and in dimensions higher than three. The criterion is given in the form of an inequality that involves integrals of a Green's function on the wetted part of submerged bodies and in this way includes the frequency of oscillations and the geometry. It is applicable for any number of bodies of arbitrary shape, subject to the external sphere condition which means that any point of the wetted surface should lie on boundary of a ball in the fluid domain.

The inequality can be checked numerically for fixed geometrical configurations and frequencies and if it is satisfied (with the accuracy of the computation taken into account) this proves uniqueness for the particular geometry and frequency. Examples of such computations will be given in this paper. In addition, estimates for the gradient of the Green function obtained in the present work allow us to obtain very simple bounds (similar to those by Simon \& Ursell (1984), but, unlike the latter, also applicable to the three-dimensional problem) for trapped modes in the space of parameters defined by submergence of the system of bodies and the frequency of oscillation; in particular, the bounds guarantee uniqueness for sufficiently large values of frequency for any system of bodies. Importantly, these simple bounds do not depend on the horizontal spacing between the bodies (for some bounds dependence on the geometry is reduced to include only the area of the wetted surface of the bodies) and, thus, for the case when the bodies are widely separated, the bounds have a significant advantage when compared with those of Simon \& Ursell (1984).

Now we give a brief outline of the paper. In section 1 we introduce notations and present the mathematical problem. A criterion of uniqueness for the problem is derived in $\S 2$. Bounds for uniqueness set in the space of frequency and submergence of bodies are obtained in $\S 3$; some examples of the bounds computed numerically are presented. Improvement of the uniqueness criterion with the help of auxiliary potentials is suggested in $\S 4$, where reduction of the criterion to a finite part of the free surface is also done. A set of auxiliary potentials is suggested in $\S 5$. Results of numerical computation for the criterion and comparison with known uniqueness results are presented in $\S 6$. The paper has two appendices where some representations and estimates for derivatives of Green's function are obtained.

\section{Statement of the problem}

We consider the linearized problem describing motion of an inviscid incompressible heavy fluid with a free surface in the presence of a system of bodies which are totally submerged to the fluid. Under the usual assumptions of linearized surface wave theory (see e.g., Lamb, 1932), the time-harmonic motion of the 
fluid can be described by a velocity potential

$$
\operatorname{Re}\{\exp (-\mathrm{i} \omega t) u(\boldsymbol{x}, y)\}
$$

where $t$ is time, $\omega$ is the frequency of the oscillations, $(\boldsymbol{x}, y)$ are spacial coordinates with the origin of the coordinate system in the unperturbed free surface, $y$ is the vertical coordinate that decreases with depth, $\boldsymbol{x}=(x, z)$ when the threedimensional problem is considered and $\boldsymbol{x}=x$ for the two-dimensional problem, corresponding to the situation of normal incidence of surface waves to a system of infinitely long cylinders with generators along the $z$-axis. The potential $u$ satisfies the equations

$$
\begin{gathered}
\Delta u=0 \quad \text { in } W, \\
\partial_{y} u-K u=0 \text { on } F,
\end{gathered}
$$

and

$$
\partial_{n} u=f \quad \text { on } \quad S .
$$

where $W$ is the domain occupied by fluid, $F=\{y=0\}$ is the mean free surface, $S$ is the wetted surface of the submerged obstruction, we use the notation $\partial_{a}^{n}=\frac{\partial^{n}}{\partial a^{n}}$ and the Laplace operator $\Delta=\partial_{x}^{2}+\partial_{y}^{2}+\partial_{z}^{2}$ in the three-dimensional case and $\Delta=\partial_{x}^{2}+\partial_{y}^{2}$ for the two-dimensional problem. Further, $K=\omega^{2} / g, g$ is the acceleration due to gravity and $\boldsymbol{n}$ is the unit normal vector directed inside the fluid. For the two-dimensional problem $W, F$ and $S$ will denote the cross-sections of the corresponding three-dimensional domains.

The standard radiation condition at infinity should also be imposed upon $u$, and the function $f$ in the last formula is defined by the type of the oscillations. We assume that $u$ belongs to $\mathrm{C}^{2}(W) \cap \mathrm{C}(\bar{W})$ and that the contours $S$ are piecewise $\mathrm{C}^{1}$-arcs and can contain corner points. The restriction on the type of corner points for which the theorems of the work are valid will be described later. We also assume that the potential $u$ has regular normal derivative at all regular points of $\partial W$.

Uniqueness of the problem is known (see e.g., Simon \& Ursell, 1984) to be equivalent to non-existence of the so-called trapped modes, that is unforced oscillations of the fluid which are adjacent to submerged obstructions and have finite energy. A trapped mode potential $u$ satisfies the conditions (1.1a), (1.1b) and, in addition,

$$
\begin{gathered}
\partial_{n} u=0 \quad \text { on } \quad S \\
\int_{W}\left\{|\nabla u|^{2}+K^{2}|u|^{2}\right\} \mathrm{d} \boldsymbol{x} \mathrm{d} y<\infty
\end{gathered}
$$

Without loss of generality, the trapped mode potential $u$ can be assumed to be real. 


\section{A criterion for uniqueness}

We start with the definition of the Green function for the three-dimensional problem. Let $G(P, Q)$ be the potential of a source located at the point $Q\left(x_{0}, y_{0}, z_{0}\right)$ and computed at the point $P(x, y, z)$. Then for $y_{0}<0$ the function $G(P, Q)$ as a function of $P$ satisfies the equations

$$
\begin{aligned}
& \left(\partial_{x}^{2}+\partial_{y}^{2}+\partial_{z}^{2}\right) G=-\delta\left(x-x_{0}, y-y_{0}, z-z_{0}\right), \quad y<0, \\
& \partial_{y} G-K u=0, \quad y=0
\end{aligned}
$$

where $\delta$ is the Dirac delta function. The function should also be bounded in the domain $y<0$ with a neighbourhood of the source $Q$ excluded.

We introduce the notations

$$
r=\left[\left|\boldsymbol{x}-\boldsymbol{x}_{0}\right|^{2}+\left(y-y_{0}\right)^{2}\right]^{1 / 2}, \quad r^{\prime}=\left[\left|\boldsymbol{x}-\boldsymbol{x}_{0}\right|^{2}+\left(y+y_{0}\right)^{2}\right]^{1 / 2},
$$

where $\boldsymbol{x}_{0}=\left(x_{0}, z_{0}\right)$. For our purposes it is immaterial which type of radiation condition at infinity is imposed upon the Green function, however it will be convenient to define the function to be real. Then, the three-dimensional Green function is known to have the following representation (see e.g. Wehausen \& Laitone, 1960)

$$
G(P ; Q)=\frac{1}{4 \pi}\left[\frac{1}{r}-\frac{1}{r^{\prime}}\right]+\frac{1}{2 \pi} \int_{0}^{\infty} \frac{\mu}{\mu-K} \mathrm{e}^{\mu\left(y+y_{0}\right)} \mathrm{J}_{0}\left(\mu\left|\boldsymbol{x}-\boldsymbol{x}_{0}\right|\right) \mathrm{d} \mu .
$$

where $J_{0}$ is the Bessel function of the first kind defined e.g. in (Gradsteyn \& Ryzhik, 1994, sect. 8.40) and the notation $f$ here and subsequently denotes the principal value integration.

Analogously, in the two-dimensional case, when $Q=Q\left(x_{0}, y_{0}\right), P=P(x, y)$, $\boldsymbol{x}=x, \boldsymbol{x}_{0}=x_{0}$ in (2.2) and the dependence in $z$ should be omitted in the conditions (2.1), we have (see e.g. Wehausen \& Laitone, 1960)

$$
G(P, Q)=\frac{1}{2 \pi}\left[\log r^{\prime}-\log r\right]+\frac{1}{\pi} \int_{0}^{\infty} \frac{\mathrm{e}^{\mu\left(y+y_{0}\right)}}{\mu-K} \cos \mu\left(x-x_{0}\right) \mathrm{d} \mu .
$$

Then, in both two- and three-dimensional problems we shall apply the third Green identity to a hypothetical trapped mode, that is a solution to the problem (1.1), and to the Green function $G$. Taking into account conditions (1.1a)-(1.1d), (2.1a) and (2.1b) we find

$$
u(Q)=\int_{S} u(P) \partial_{n(P)} G(P, Q) \mathrm{d} s_{(P)}, \quad Q \in W .
$$

From the latter formula it follows that

$$
|u(Q)| \leqslant \max _{P \in S}\{|u(P)|\} \int_{S}\left|\partial_{n(P)} G(P, Q)\right| \mathrm{d} s_{(P)} .
$$


We note that the Green function can be extended analytically from the domain $y<0$ to the domain $y<-y_{0}$ (it follows in particular from the representations (2.3) and (2.4)). Thus, in the case under consideration, when bodies are submerged, the representation (2.5) is clearly valid for a point $Q$ belonging to the free surface. Thus, from (2.6) we arrive at the inequality

$$
\sup _{F}|u| \leqslant \max _{S}|u| \sup _{Q \in F}\left\{\int_{S}\left|\partial_{n(P)} G(P, Q)\right| \mathrm{d} s_{(P)}\right\} .
$$

Further we can make use of maximum principles to compare the values $\sup _{F}|u|$ and $\max _{S}|u|$. The strong maximum principle of E. Hopf (see e.g., Gilbarg \& Trudinger, 1983, Theorem 3.5) guarantees that a potential $u$ satisfying (1.1a) cannot attain a minimum or maximum at an interior point of any finite subset of $W$. The theorem is also valid for the unbounded domain $W$, we should only note that under the condition (1.1d) the value of $|u|$ decays at infinity (see the expansions at infinity derived by Ursell (1950)). Furthermore, Lemma 3.4 from (Gilbarg \& Trudinger, 1983) is applicable and this states that any outward directional derivative at a boundary point where $u$ attains its maximum (minimum) is positive (negative). In view of the condition (1.1c), the latter guarantees that the maximum/minimum is located in $F$ but not in $S$. Use of the latter assertion imposes an additional restriction on the geometry of the contour $S$ known as the exterior sphere condition, this requires that the point $Q$ of maximum/minimum should lie on the boundary of a ball in $W$.

We note that if $u$ is constant, then $u=0$ by (1.1b). Thus, if the geometrical restriction on $S$ is satisfied, we find that either $u \equiv 0$ in $W$ or

$$
\max _{S}|u|<\sup _{F}|u| .
$$

Combining the inequalities (2.7) and (2.8) we arrive at the following assertion.

THEOREM 2.1: If for a given geometry $S$ and a given value of the parameter $K$ the inequality

$$
\sup _{Q \in F}\left\{\int_{S}\left|\partial_{n(P)} G(K ; P, Q)\right| \mathrm{d} s_{(P)}\right\} \leqslant 1
$$

holds, then the problem (1.1) has only the trivial solution for this geometry and with the particular value of the parameter $K$.

\section{Uniqueness bounds in frequency and submergence}

Consider a fixed system of bodies $S(Y)$ located at the depth

$$
Y=\max \{y:(\boldsymbol{x}, y) \in S\}, \quad Y<0 .
$$

We can use the representation (A.8) and estimates (A.9) and (B.17) to find a subset $\Omega$ of the $(K, Y)$ parameter space such that uniqueness of the problem 


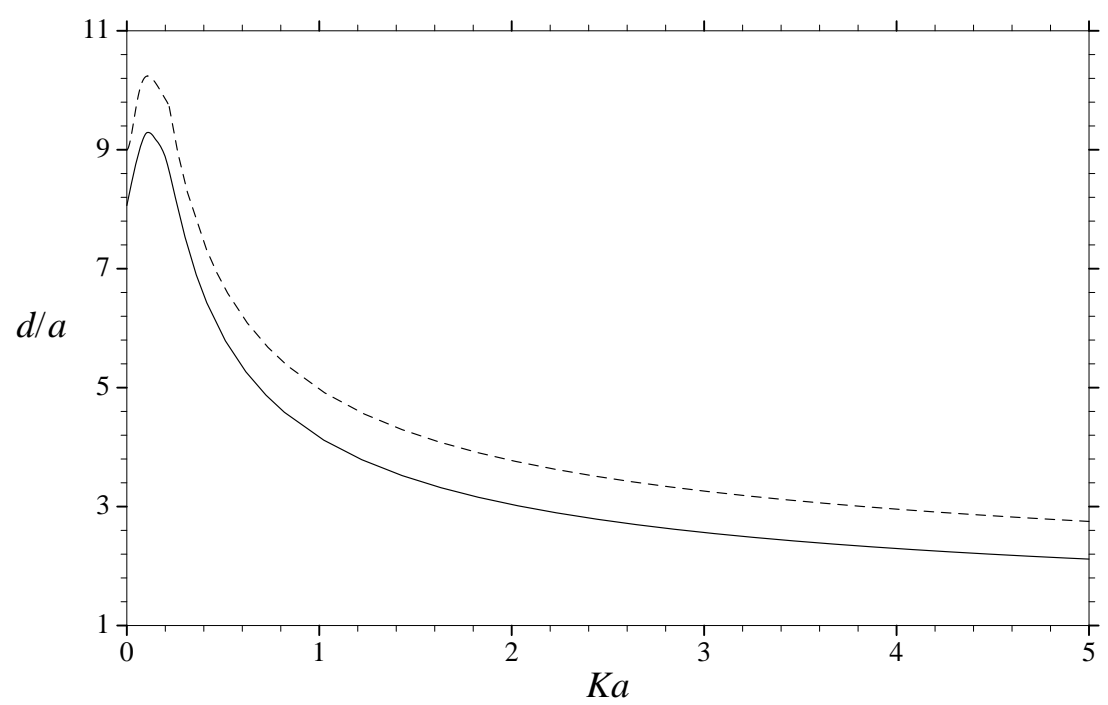

Figure 1: Bounds given by the formulae (3.4) (solid line) and (3.5) (dashed line) for two circular cylinders. The uniqueness domain is located above the curves.

(1.1) is guaranteed in $\Omega$. We start with the criterion (2.9) and write

$$
\begin{aligned}
\int_{S}\left|\partial_{n(\boldsymbol{x}, y)} G\left(\boldsymbol{x}, y, \boldsymbol{x}_{0}, 0\right)\right| \mathrm{d} s_{(\boldsymbol{x}, y)} & \leqslant \int_{S}\left|\nabla_{\boldsymbol{x}, y} G\left(\boldsymbol{x}, y, \boldsymbol{x}_{0}, 0\right)\right| \mathrm{d} s_{(\boldsymbol{x}, y)} \\
& \leqslant \int_{S} g(K, y) \mathrm{d} s_{(\boldsymbol{x}, y)}
\end{aligned}
$$

where by (B.17) for the three-dimensional case

$$
g(K, y)=K^{2}\left(\frac{1}{\pi^{2}}+\frac{1}{4}\right)^{1 / 2}\left[\mathrm{e}^{2 K y}+\pi^{-2} \min \left\{\frac{8}{(K y)^{4}}, \frac{100}{(K y)^{6}}\right\}\right]^{1 / 2},
$$

and by (A.8) and (A.9) for the two-dimensional case

$$
g(K, y)=K\left[\mathrm{e}^{2 K y}+\pi^{-2} \min \left\{\frac{4}{(K y)^{2}}, \frac{(2 \sqrt{2}+1)^{2}}{(K y)^{4}}\right\}\right]^{1 / 2} .
$$

In each case the function $g(K, y)$ is monotonically decreasing in the depth $y$.

Thus, the boundary of the region in the $(K, Y)$ parameter space for which uniqueness is guaranteed is given by

$$
\int_{S(Y)} g(K, y) \mathrm{d} s_{(\boldsymbol{x}, y)}=1
$$

a weaker condition is

$$
\sum_{i=1}^{N} g\left(K, Y_{i}\right)\left|S_{i}\right|=1
$$




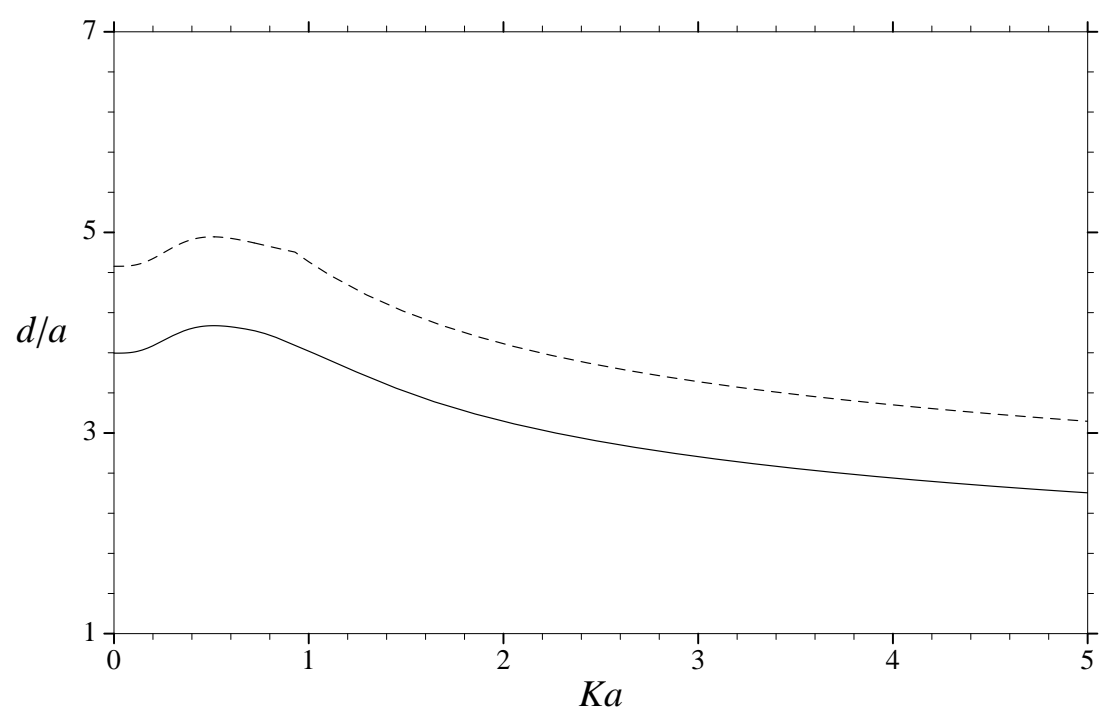

Figure 2: Bounds given by the formulae (3.4) (solid line) and (3.5) (dashed line) for two spheres. The uniqueness domain is located above the curves.

where $S_{i}$ is one of the bodies forming the $N$-body system $S,\left|S_{i}\right|$ is the area of its surface, and $Y_{i}=\max \left\{y:(\boldsymbol{x}, y) \in S_{i}\right\}$.

For any system of bodies of given submergence and geometry uniqueness is guaranteed by (2.9) and (3.1) for sufficiently large values of $K \geqslant K^{*}$. This follows from the estimates $O\left(K^{-1}\right)$ as $K \rightarrow \infty$ for the functions $g$ defined in (3.2) and (3.3). The bound $K^{*}$ specific to the geometry and submergence of the bodies, but not depending of their relative horizontal positions, can be found from (3.4) or (3.5). It is notable that, unlike the bounds obtained by Simon \& Ursell (1984) and Kuznetsov \& Simon (1999), the bounds obtained from (3.4) and (3.5) do not depend on the horizontal spacing between the bodies, and, thus, give an improvement when the spacing is large. This will be seen later in $\S 6$, where the bounds (3.4) and the bounds of Kuznetsov \& Simon (1999) are compared.

Results of numerical evaluation of the curves in parameter space given by (3.4) and (3.5) are shown in fig. 1 and 2. The computations are for a system consisting of two circles (fig. 1) and two spheres (fig. 2) of radius $a$ and whose centres are located at a distance $l$ from the line $\boldsymbol{x}=0$ and at a depth $d$. Non-existence of trapped modes for the geometry is guaranteed in the sets of parameters located in fig. 1 and 2 above the curves.

The asymptotic behaviour for small and large $K a$ of the curves in figures 1 and 2 can be obtained as follows. Consider first of all the two-dimensional case shown in fig. 1. From (3.3), as $K a \rightarrow 0$

$$
g(K, y) \sim \frac{2}{\pi|y|}
$$


and therefore

$$
\int_{S(Y)} g(K, y) \mathrm{d} s_{(\boldsymbol{x}, y)} \sim \frac{4 a}{\pi} \int_{0}^{2 \pi} \frac{\mathrm{d} \theta}{d-a \sin \theta}=\frac{8}{\left((d / a)^{2}-1\right)^{1 / 2}}
$$

so that (3.4) holds provided $d / a \rightarrow \sqrt{65}$ as $K a \rightarrow 0$. In the same limit

$$
\sum_{i=1}^{2} g\left(K, Y_{i}\right)\left|S_{i}\right| \sim \frac{8}{d / a-1}
$$

and, thus, $d / a \rightarrow 9$ as $K a \rightarrow 0$ for (3.5). It may be noted that since the estimates (3.4), (3.5) in terms of the functions (3.2) and (3.3) are based on the expansion (A.6) of the exponential integral and this expansion is most suited for large values of the argument (see Appendices), we cannot expect the estimates (3.4) and (3.5) to be effective for small values of $K$, and indeed $d / a$ has a finite limit as $K a \rightarrow 0$. However, the theorem of Simon \& Ursell (1984) guarantees (in the two-dimensional case) uniqueness for any submergence when $K a$ is sufficiently small or large.

Further, as $K a \rightarrow \infty,(3.3)$ gives

$$
g(K, y) \sim \frac{2 \sqrt{2}+1}{\pi K y^{2}}
$$

and therefore

$$
\int_{S(Y)} g(K, y) \mathrm{d} s_{(\boldsymbol{x}, y)} \sim \frac{2(2 \sqrt{2}+1) a}{\pi K} \int_{0}^{2 \pi} \frac{\mathrm{d} \theta}{(d-a \sin \theta)^{2}}=\frac{4(2 \sqrt{2}+1) d / a}{K a\left((d / a)^{2}-1\right)^{3 / 2}} .
$$

For the last expression to be non-zero in the limit $K a \rightarrow \infty$, it is required that $d / a \rightarrow 1$ and hence

$$
\frac{4(2 \sqrt{2}+1) d / a}{K a\left((d / a)^{2}-1\right)^{3 / 2}} \sim \frac{4(1+1 /(2 \sqrt{2}))}{K a(d / a-1)^{3 / 2}},
$$

so that (3.4) holds provided

$$
\frac{d}{a} \sim 1+\left(\frac{4(1+1 /(2 \sqrt{2}))}{K a}\right)^{2 / 3} \text { as } K a \rightarrow \infty
$$

As $K a \rightarrow \infty$

$$
\sum_{i=1}^{2} g\left(K, Y_{i}\right)\left|S_{i}\right| \sim \frac{4(2 \sqrt{2}+1)}{K a(d / a-1)^{2}}
$$

and (3.5) holds provided

$$
\frac{d}{a} \sim 1+2\left(\frac{2 \sqrt{2}+1}{K a}\right)^{1 / 2} \text { as } K a \rightarrow \infty .
$$


Similar calculations may be made for the asymptotics of the three-dimensional case shown in fig. 2 and in view of (3.2) the results are as follows. As $K a \rightarrow 0$, (3.4) and (3.5) give

$$
\frac{d}{a} \rightarrow\left[1+16 \sqrt{2}\left(\frac{1}{\pi^{2}}+\frac{1}{4}\right)^{1 / 2}\right]^{1 / 2}, \quad \frac{d}{a} \rightarrow 1+4\left[2\left(\frac{1}{\pi^{2}}+\frac{1}{4}\right)\right]^{1 / 4}
$$

respectively. As $K a \rightarrow \infty$ (3.4) and (3.5) hold provided

$$
\frac{d}{a} \sim 1+2\left(\frac{5}{K a}\right)^{1 / 2}\left(\frac{1}{\pi^{2}}+\frac{1}{4}\right)^{1 / 4}, \quad \frac{d}{a} \sim 1+2\left[\frac{10}{K a}\left(\frac{1}{\pi^{2}}+\frac{1}{4}\right)^{1 / 2}\right]^{1 / 3}
$$

respectively. Similar asymptotic analysis can be done for bodies of arbitrary shape.

\section{Modification of the uniqueness criterion}

In the section we shall show how the uniqueness result of Theorem 2.1 can be improved. Suppose we can find potentials $\Phi_{i}$ defined and bounded on $W$ and satisfying the equations (1.1a) and (1.1b). Then, by Green's identity (provided that the potentials and their derivatives have the behaviour at infinity that is required for the application of the identity) we find

$$
0=\int_{S} u(P) \partial_{n} \Phi_{i}(P) \mathrm{d} s
$$

Combining the latter with (2.5) we get

$$
u(Q)=\int_{S} u(P)\left[\partial_{n(P)} G(P, Q)+\sum_{i} c_{i} \partial_{n} \Phi_{i}(P, Q)\right] \mathrm{d} s_{(P)}, \quad Q \in W \cup F,
$$

where $c_{i}$ are arbitrary constants and we indicate explicitly that $\Phi_{i}$ can also contain dependence on $Q$. We fix the functions $c_{i}=c_{i}(Q)$ as solutions to the minimization problem

$$
\min _{c_{1}, c_{2}, \ldots} \int_{S}\left|\partial_{n(P)} G(P, Q)+\sum_{i} c_{i} \partial_{n} \Phi_{i}(P, Q)\right| \mathrm{d} s_{(P)}, \quad Q \in F
$$

and from (4.1) arrive at the inequality

$$
\sup _{F}|u| \leqslant \max _{S}|u| \sup _{Q \in F}\left\{\int_{S}\left|\partial_{n(P)} G(P, Q)+\sum_{i} c_{i}(Q) \partial_{n} \Phi_{i}(P, Q)\right| \mathrm{d} s_{(P)}\right\} .
$$

which, along with the maximum principles, proves the assertion 
O.V. Motygin, P. McIver: Uniqueness in problems of wave-body interaction

THEOREM 4.1: If for a given geometry $S$ and a value of the parameter $K$ the inequality

$\mathcal{M}\left(F, S,\left\{\Phi_{i}\right\}\right) \equiv \sup _{Q \in F}\left\{\int_{S}\left|\partial_{n(P)} G(P, Q)+\sum_{i} c_{i}(Q) \partial_{n} \Phi_{i}(P, Q)\right| \mathrm{d} s_{(P)}\right\} \leqslant 1$

holds, where the functions $c_{i}(Q)$ are defined by (4.2), then the problem (1.1) has only the trivial solution.

The minimization problem (4.2) is a problem of linear programming and can be effectively solved numerically by the simplex method. More details will be given below in the section 6 .

The supremum in the formula (4.3) is sought in the infinite set $F$, which is inappropriate for numerical application of the criterion. Therefore, it is important to note that the computation can be reduced to a finite set, because such a finite part of the free surface $F_{0}$ can be found that the expression $\mathcal{M}\left(F \backslash F_{0}, S,\left\{\Phi_{i}\right\}\right)$ for the rest of the free surface it is guaranteed to be smaller than one.

Consider the expression (4.2) in the two-dimensional case for a point in the free surface $Q=\left(x_{0}, 0\right)$. Let the point $Q$ be located on the right side from the bodies, so that $x \leqslant x_{0}$ for all $P(x, y) \in S$. Then, by (A.8) we have

$$
\begin{aligned}
& \partial_{x} G(P, Q)=K \mathrm{e}^{K y} \cos K\left(x-x_{0}\right)-K \pi^{-1} \operatorname{Im}\left\{R_{1}(\mathrm{i} K \zeta)\right\}, \\
& \partial_{y} G(P, Q)=K \mathrm{e}^{K y} \sin K\left(x-x_{0}\right)-K \pi^{-1} \operatorname{Re}\left\{R_{1}(\mathrm{i} K \zeta)\right\},
\end{aligned}
$$

where $\zeta=x-x_{0}+\mathrm{i} y$ and $R_{1}$ is given in (A.7).

Then, one can find by the particular choice $c_{1}=-1, \Phi_{1}=\mathrm{e}^{K y} \sin K\left(x-x_{0}\right)$ and $c_{i}=0, i \neq 1$, in (4.3) that

$$
\begin{aligned}
& \min _{c_{1}, c_{2}, \ldots} \int_{S}\left|\partial_{n(P)} G(P, Q)+\sum_{i} c_{i} \partial_{n(P)} \Phi_{i}(P, Q)\right| \mathrm{d} s_{(P)} \\
& \leqslant \int_{S}\left|\partial_{n(P)}\left(G(P, Q)-\mathrm{e}^{K y} \sin K\left(x-x_{0}\right)\right)\right| \mathrm{d} s_{(P)} \\
& \quad \leqslant \int_{S} R_{G}\left(\left|x-x_{0}\right|, y\right) \mathrm{d} s_{(x, y)},
\end{aligned}
$$

where the function $R_{G}$ is defined in (A.9).

Let the inequality

$$
\int_{S} R_{G}\left(\left|x-x_{0}\right|, y\right) \mathrm{d} s_{(x, y)}<1,
$$

hold for $x_{0}=a_{+}^{*}$ and $x_{0}=a_{-}^{*}$, where

$$
a_{+}^{*} \geqslant \max \{x:(x, y) \in S\}, \quad a_{-}^{*} \leqslant \min \{x:(x, y) \in S\} .
$$

Since the function $R_{G}\left(\left|x-x_{0}\right|, y\right)$ decays monotonically in $\left|x-x_{0}\right|$, the inequalities (4.5) hold for all $x_{0} \in F$, such that $\pm x_{0}> \pm a_{ \pm}^{*}$. Then, the inequalities (4.4) and (4.5) guarantees that

$$
\mathcal{M}\left(F \backslash F_{0}, S,\left\{\Phi_{i}\right\}\right)<1, \quad F_{0}=\left\{(x, 0): a_{-}^{*} \leqslant x \leqslant a_{+}^{*}\right\}
$$


and to establish uniqueness using the criterion of Theorem 4.1 we have only to check the corresponding inequality over the finite part of the free surface $F_{0}$.

In the three-dimensional case it is much more obvious that the criterion can be evaluated in a finite part of the free surface because the Green function and its gradient decay at infinity (see e.g. Kuznetsov et al., 2002, part I) as $O\left(\left|\boldsymbol{x}-\boldsymbol{x}_{0}\right|^{-1 / 2}\right)$. A better result can be obtained by extracting simple waves in the way it has been done above for the two-dimensional problem, but such analysis is beyond the scope of this work.

\section{Auxiliary potentials}

In this section we suggest a set of auxiliary potentials $\Phi_{i}$. Consider first the twodimensional problem. The simplest auxiliary potentials are the wave solutions, which have been used in the previous section,

$$
\widetilde{\Phi}^{(+)}(P, Q)=\mathrm{e}^{K\left(y-y_{0}\right)} \cos K\left(x-x_{0}\right), \quad \widetilde{\Phi}^{(-)}(P, Q)=\mathrm{e}^{K\left(y-y_{0}\right)} \sin K\left(x-x_{0}\right) .
$$

Introduce two polar coordinate systems $(r, \theta)$ and $(r, \theta)$, where $r$ and $r^{\prime}$ are given by (2.2) and $\theta$ and $\theta^{\prime}$ are such that

$$
x-x_{0}=r \sin \theta=r^{\prime} \sin \theta^{\prime}, \quad y=r \cos \theta+y_{0}=-r^{\prime} \cos \theta^{\prime}-y_{0} .
$$

Using the notation we write the following multipole solutions, satisfying (1.1a) and (1.1b),

$$
\begin{aligned}
\Phi_{n}(P, Q)=\frac{\mathrm{e}^{-\mathrm{i} n \theta}}{r^{n}}+\frac{(-1)^{n}}{(n-1) !} \int_{0}^{\infty} \frac{\mu+K}{\mu-K} \mu^{n-1} \mathrm{e}^{\mu\left(y+y_{0}\right)} \mathrm{e}^{\mathrm{i} \mu\left(x-x_{0}\right)} \mathrm{d} \mu, \\
n=1,2, \ldots, \quad \Phi_{0}(P, Q)=G(P, Q) .
\end{aligned}
$$

Let $Q_{j}, j=0,1,2, \ldots$, be a system of points located inside the contours $S$. Then, obviously, the real and imaginary parts of $\Phi_{n}\left(P, Q_{j}\right)$ can be used as auxiliary potentials.

However, numerical computation of the potentials (5.2) is a difficult task due to the integration of oscillatory functions with singularities, which should be performed over the infinite interval. Hence, much more convenient objects for numerical computation are the auxiliary potentials based on the wave-free potentials

$$
\Psi_{i}^{(+)}(P)=\operatorname{Re}\left\{\Psi_{n_{i}+1}\left(P, Q_{j_{i}}\right)\right\}, \quad \Psi_{i}^{(-)}(P)=\operatorname{Im}\left\{\Psi_{n_{i}+1}\left(P, Q_{j_{i}}\right)\right\},
$$

where $n_{i}, j_{i} \in\{0,1, \ldots\}$ and for $n=1,2,3, \ldots$

$$
\Psi_{n}(P, Q)=K \frac{\mathrm{e}^{-\mathrm{i} n \theta}}{r^{n}}-K \frac{\mathrm{e}^{-\mathrm{i} n \theta^{\prime}}}{r^{\prime n}}-n \frac{\mathrm{e}^{-\mathrm{i}(n+1) \theta}}{r^{n+1}}-n \frac{\mathrm{e}^{-\mathrm{i}(n+1) \theta^{\prime}}}{r^{\prime n+1}}
$$

(see e.g. Eatock Taylor \& Hu, 1991). 
The expansion theorem of Ursell (1950) states that any wave potential can be written at great distances as the sum of a wave source, a horizontal wave dipole, regular waves and wave-free potentials and, thus, at least formally, substantiates choosing auxiliary potentials from the set $\left\{\widetilde{\Phi}^{ \pm}, \Phi_{0}, \operatorname{Im} \Phi_{1}, \Psi_{1}^{ \pm}, \Psi_{2}^{ \pm}, \ldots\right\}$.

Now consider the three-dimensional problem. We introduce cylindrical coordinate system with its origin at the point $Q\left(x_{0}, y_{0}, z_{0}\right)$, where the radius $R$ and the angular coordinate $\alpha$ for the point $P(x, y, z)$ are defined by

$$
R=\left|\boldsymbol{x}-\boldsymbol{x}_{0}\right|, \quad x-x_{0}=R \cos \alpha, \quad z-z_{0}=R \sin \alpha .
$$

Then, it is easy to check that the functions

$$
\widetilde{\Phi}_{m}(P, Q)=\mathrm{e}^{K\left(y-y_{0}\right)} \mathrm{J}_{m}(K R)\left\{\begin{array}{c}
\cos m \alpha \\
\sin m \alpha
\end{array}\right\}, \quad m=0,1,2, \ldots
$$

satisfy the Laplace equation (1.1b) and the condition on the free surface (1.1b), thus they can be applied in the criterion (4.3).

Similar to the two-dimensional case, more potentials $\Phi$ for application in (4.3) could be constructed using multipole singular solutions of Laplace's equation (for their definition see e.g., Wehausen \& Laitone, 1960) with the singular points located inside the contours $S$. However, for effective computation the following system is preferable (the points $Q_{j}$ are located inside the contours $S$ )

$$
\Phi_{i}(P)=\Psi_{\ell_{i}}^{m_{i}}\left(P, Q_{j_{i}}\right), \quad j_{i}, \ell_{i}, m_{i}=0,1,2, \ldots
$$

which is based on the following wave-free potentials (see Wehausen \& Laitone, 1960, 13.21)

$$
\begin{aligned}
\Psi_{\ell}^{m}(P, Q)= & {\left[\frac{P_{\ell+1}^{m}(-\cos \theta)}{r^{\ell+2}}-\frac{K}{\ell-m+1} \frac{P_{\ell}^{m}(-\cos \theta)}{r^{\ell+1}}\right.} \\
& \left.+(-1)^{m} \frac{P_{\ell+1}^{m}\left(\cos \theta^{\prime}\right)}{r^{\ell+2}}+\frac{(-1)^{m} K}{\ell-m+1} \frac{P_{\ell}^{m}\left(\cos \theta^{\prime}\right)}{r^{\prime \ell+1}}\right]\left\{\begin{array}{l}
\cos m \alpha \\
\sin m \alpha
\end{array}\right\} .
\end{aligned}
$$

where $\ell, m=0,1,2, \ldots, P_{\ell}^{m}$ are associated Legendre functions (see e.g. 8.752(1) in Gradsteyn \& Ryzhik (1994), 8.6.6 in Abramowitz \& Stegun (1965)).

\section{Numerical results}

In this section we shall give examples of numerical evaluation of the criterion (4.3). Numerical investigation was performed for the criterion (4.3) in the two-dimensional case with the minimisation (4.2) implemented using the NAG (Numerical Algorithms Group) routine E04CCF which is based on the simplex method (see the NAG web site at www.nag.co.uk for details of this routine). As in $\S 3$ we consider a system consisting of two circles of radius $a$ whose centres are located at a distance $l$ from the line $x=0$ and at a depth $d$. Results of computation for the criterion (4.3) are presented in fig. 3 and 4 for $d / a=2$ 


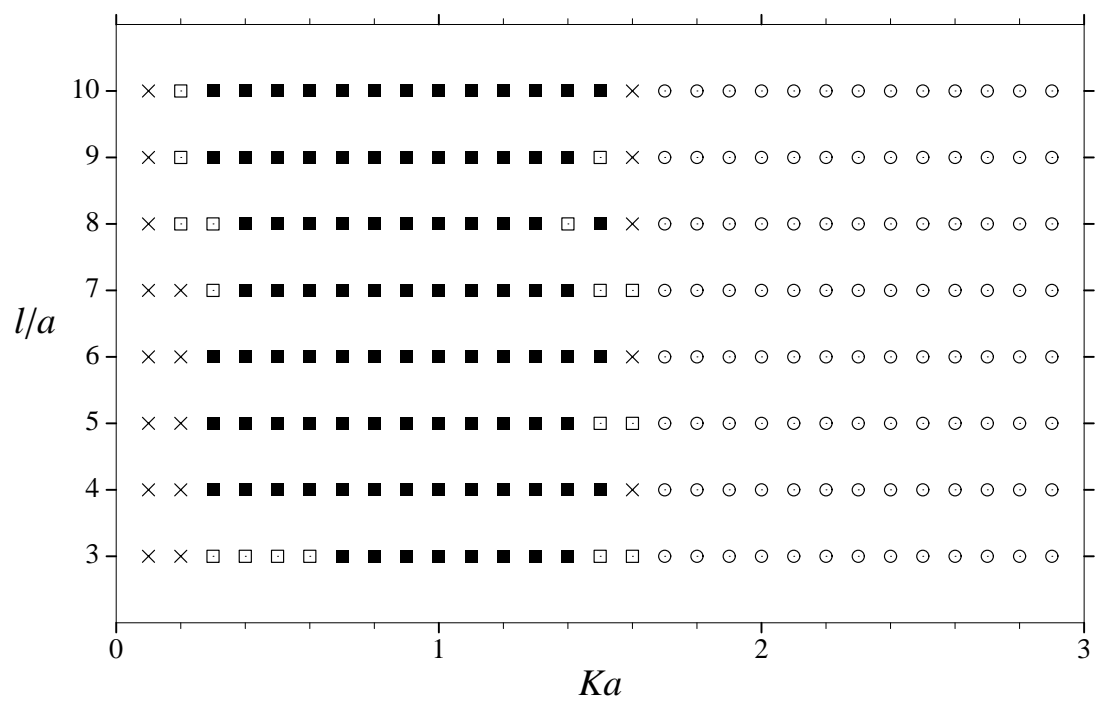

Figure 3: Results of numerical investigation of criterion (4.3) for two cylinders with $d / a=2$.

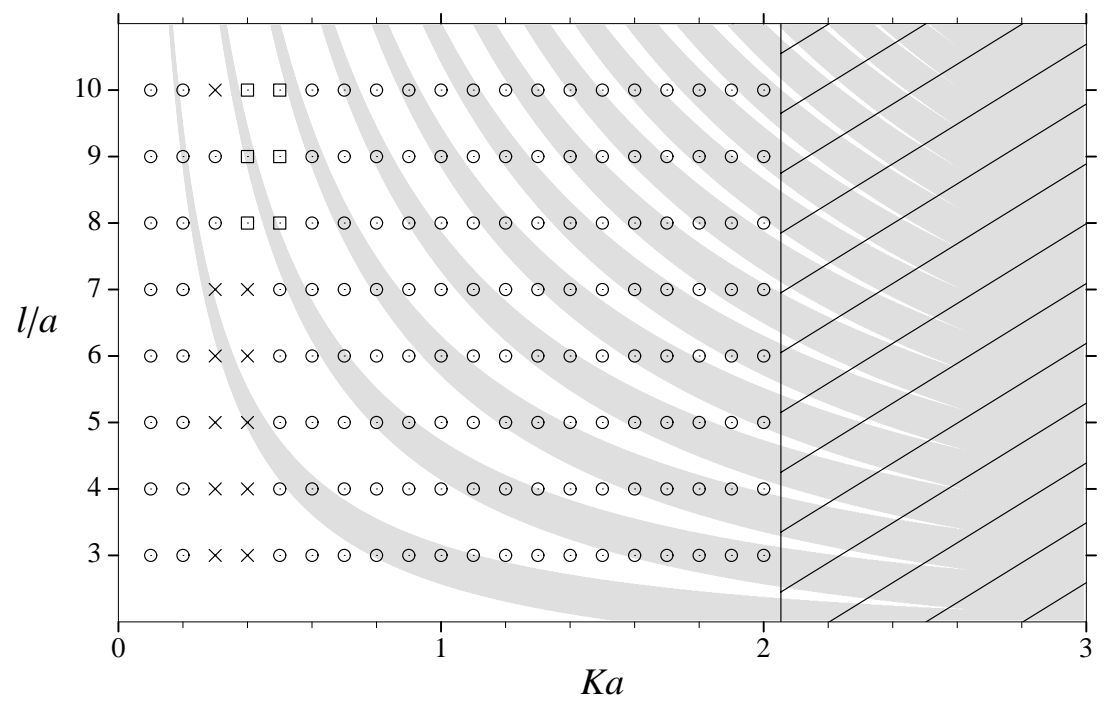

Figure 4: Results of numerical investigation of criterion (4.3) for two cylinders with $d / a=3$. In the grey area uniqueness is given by criterion of Kuznetsov \& Simon (1999); the hatched area of uniqueness is defined by the bound (3.4)

and $d / a=3$ respectively. The notations $\circ, \times$ and $\square$ are used for the points where non-existence of trapped modes is shown using (4.3). The mark o means that no auxiliary potentials are needed, at the points $\times$ the non-existence of trapped modes is established using the auxiliary simple-wave potentials (5.1) and $\square$ means that both simple waves and four waveless potentials (5.3) are involved to improve the criterion. The points marked by the symbols $\mathbf{m}$ indicate where the criterion (4.3) could not be satisfied using any of the above-mentioned auxiliary potentials; calculations with an increased number of auxiliary potentials failed 
due to difficulties in finding a minimum in (4.2). Uniqueness in the hatched region of fig. 4 is guaranteed by (3.1) and (3.3); with the parameters of fig. 3 the corresponding region of uniqueness is for $K a$ greater than about 5.900.

In fig. 4 the results of our computations are compared with results of Kuznetsov \& Simon (1999) which are only applicable to symmetric geometries (that work is unable to make predictions about the case shown in fig. 3). The grey areas in fig. 4 correspond to the domain where uniqueness is guaranteed for both symmetric and antisymmetric modes by the criterion of Kuznetsov \& Simon (1999).

\section{Conclusion}

In this paper the question of uniqueness for the two and three-dimensional linearized boundary-value problems of wave-body interaction is considered in the case when the fluid has an unbounded free surface and the bodies are totally submerged. A new criterion for uniqueness is suggested, which is based on Green's integral identity and maximum principles for elliptic differential equations. The criterion is given in the form of an inequality that involves integrals of Green's function on the wetted part of submerged bodies (in this way including the frequency of oscillations and the geometry). The integrals can also contain auxiliary potentials and a set of such potentials is suggested for improvement of the result. The criterion can be checked numerically for fixed geometrical configurations and frequencies and if it is satisfied (with the accuracy of the computation taken into account) this proves uniqueness for the particular geometry and frequency. Examples of such computations are given. In addition, simple bounds are also derived from the criterion, which deliver uniqueness sets in the space of parameters defined by submergence of the system of bodies and the frequency of oscillation. It is noteworthy that these bounds do not depend on the horizontal spacing between the bodies (for some bounds dependence on the geometry is reduced to include only the area of the wetted surface of the bodies) and, thus, for the case when the bodies are widely separated, the bounds have a significant advantage when compared with those of Simon \& Ursell (1984) or Kuznetsov \& Simon (1999) (the latter bounds are for symmetric structures). The suggested criterion is applicable to any number of bodies of arbitrary shape, subject to the external sphere condition, and can also be generalised for more complicated elliptic problems. It seems very likely that an improvement of the criterion can be achieved for symmetric structures; in particular, for the twodimensional problem symmetric and antisymmetric in $x$ modes can be considered separately. Perhaps the simplest further applications of the suggested criterion could be to the problem in finite-depth fluid and to the two-dimensional problem describing oblique incidence of waves to cylindrical structures where in the set of equations (1.1) the field equation is replaced by $\Delta u-k^{2} u=0$. 


\section{Acknowledgement}

The first author would like to acknowledge the financial support by Royal Society/NATO/FCO Chevening under a Postdoctoral Fellowship Award grant, and the support of his visit to Loughborough University by London Mathematical Society and Russian Foundation of Basic Research.

\section{References}

Abramowitz, M., Stegun, I.A., (1965). Handbook of mathematical functions, Dover, New York, 1046 pp.

Eatock Taylor, R., Hu, C.S., (1991). Multipole expansions for wave diffraction and radiation in deep water, Ocean Engng., 18(3), 191-224.

Gradsteyn, I.S., Ryzhik, I.M., (1994). Table of integrals, series and products. 5th edition, Academic Press, New York, 1204 pp.

Gilbarg, D., Trudinger, N.S., (1983). Elliptic Partial Differential Equations of Second Order, Springer-Verlag, Berlin Heidelberg New York, 524 pp.

Hulme, A., (1984). Some applications of Maz'ja's uniqueness theorem to a class of linear water wave problems, Math. Proc. Camb. Phil. Soc., 95, 511-519.

John, F., (1950). On the motion of floating bodies, II, Comm. Pure Appl. Math., 3(1), 45-101.

Kuznetsov, N., Maz'ya, V., Vainberg, B., (2002). Linear Water Waves: A Mathematical Approach, Cambridge University Press, Cambridge, 512 pp.

Kuznetsov, N.G., Simon, M.J., (1999). A note on uniqueness in the linearized waterwave problem, J. Fluid Mech., 386, 5-14.

Lamb, H., (1932). Hydrodynamics. 6th edition, Cambridge University Press, Cambridge, 738 pp.

Maz'ya, V.G., (1978). Solvability of the problem on the oscillations of a fluid containing a submerged body, J. Soviet Math., 10, 86-89.

McIver, M., (1996). An example of non-uniqueness in the two-dimensional linear water wave problem, J. Fluid Mech., 315, 257-266.

McIver M., (2000). Trapped modes supported by submerged obstacles, Proc. Roy. Soc. London A, 456, 1851-1860.

McIver, M., Porter, R., (2002). Trapping of waves by a submerged elliptical torus, J. Fluid Mech., 456, 277-293.

Porter, R., (2002). Trapping of water waves by pairs of submerged cylinders, Proc. Roy. Soc. London A, 458, 607-624.

Simon, M.J., Ursell, F., (1984). Uniqueness in linearized two-dimensional water-wave problems, J. Fluid Mech., 148, 137-154.

Ursell, F., (1950). Surface waves on deep water in the presence of a submerged circular cylinder, II, Proc. Camb. Phil. Soc., 46, 153-158. 
Ursell, F., (1992). Some unsolved and unfinished problems in the theory of waves, Wave Asymptotics, P.A.Martin, G.R.Wickham, Cambridge University Press, 220244.

Vainberg, B.R., Maz'ya, V.G., (1973). On the problem of the steady-state oscillations of a fluid layer of variable depth, Trans. Moscow Math. Soc., 28, 56-73. (translated from Russian)

Wehausen, J.V., Laitone, E.V., (1960). Surface waves, Handbuch der Physik, 9, 446-778. Springer, Berlin.

\section{Appendix A. On derivatives of the two-dimensional Green function}

In the appendix we shall derive estimates for derivatives of the function $G(P, Q)$ in the case when the source point $Q$ belongs to the free surface $F$. From the representation (2.4) we have

$$
G\left(x, y, x_{0}, 0\right)=\frac{1}{\pi} \int_{0}^{\infty} \frac{\mathrm{e}^{\mu y} \cos \mu\left(x-x_{0}\right)}{\mu-K} \mathrm{~d} \mu .
$$

Then, we find

$$
\begin{aligned}
\partial_{x} G\left(x, y, x_{0}, 0\right) & =-\frac{1}{\pi} \int_{0}^{\infty} \mathrm{e}^{\mu y} \sin \mu\left(x-x_{0}\right) \mathrm{d} \mu-\frac{K}{\pi} \int_{0}^{\infty} \frac{\mathrm{e}^{\mu y} \sin \mu\left(x-x_{0}\right) \mathrm{d} \mu}{\mu-K} \\
& =-\frac{x-x_{0}}{\pi\left[\left(x-x_{0}\right)^{2}+y^{2}\right]}-\frac{K}{\pi} \operatorname{Im}\left\{\mathrm{e}^{-\mathrm{i} K \zeta} \operatorname{Ei}(\mathrm{i} K \zeta)\right\} \\
\partial_{y} G\left(x, y, x_{0}, 0\right) & =-\frac{y}{\pi\left[\left(x-x_{0}\right)^{2}+y^{2}\right]}+\frac{K}{\pi} \int_{0}^{\infty} \frac{\mathrm{e}^{\mu y} \cos \mu\left(x-x_{0}\right) \mathrm{d} \mu}{\mu-K} \\
& =-\frac{K}{\pi\left[\left(x-x_{0}\right)^{2}+y^{2}\right]}-\frac{K}{\pi} \operatorname{Re}\left\{\mathrm{e}^{-\mathrm{i} K \zeta} \operatorname{Ei}(\mathrm{i} K \zeta)\right\}
\end{aligned}
$$

where $\zeta=x-x_{0}+\mathrm{i} y$ and $\mathrm{Ei}$ is the integral exponent (see 8.212.5 in Gradsteyn \& Ryzhik (1994))

$$
\operatorname{Ei}(z)=-\mathrm{e}^{z} \int_{0}^{\infty} \frac{\mathrm{e}^{-z t} \mathrm{~d} t}{t-1}, \quad \operatorname{Re}\{z\}>0 .
$$

It will be convenient to use the following form of the formulae (A.1) and (A.2)

$$
\partial_{x} G-\mathrm{i} \partial_{y} G=\frac{\mathrm{i} K}{\pi}\left[-\frac{1}{\mathrm{i} K \zeta}+\mathrm{e}^{-\mathrm{i} K \zeta} \operatorname{Ei}(\mathrm{i} K \zeta)\right] .
$$

Now we derive an expansion of the function Ei. We write

$$
f_{0}^{\infty} \frac{\mathrm{e}^{-z t} \mathrm{~d} t}{t-1}=f_{0}^{\infty} \frac{\mathrm{e}^{-z t} \mathrm{~d} t}{t-1}+\pi \mathrm{ie}^{-z}
$$


where $f$ means that the line of integration passes above the pole. Moving the contour of integration we have

$$
\int_{0}^{\infty} \frac{\mathrm{e}^{-z t} \mathrm{~d} t}{t-1}-\pi \mathrm{ie}^{-z}=\int_{\ell^{+}} \frac{\mathrm{e}^{-z t} \mathrm{~d} t}{t-1}
$$

where $\ell^{+}$is the ray emanating from the origin and going to infinity through the point $-(\mathrm{i} z)^{-1}$. Then, by change of variable $t=\tau / \mathrm{i} z$ we obtain

$$
\int_{\ell^{+}} \frac{\mathrm{e}^{-z t} \mathrm{~d} t}{t-1}=\int_{0}^{-\infty} \frac{\mathrm{e}^{\mathrm{i} \tau} \mathrm{d} \tau}{\tau-\mathrm{i} z}=\int_{0}^{+\infty} \frac{\mathrm{e}^{\mathrm{i} \tau} \mathrm{d} \tau}{\tau-\mathrm{i} z}-2 \pi \mathrm{ie}^{-z}
$$

Let $\operatorname{Im}\{z\}>0$. Integrating by parts $N$ times we arrive at

$$
\int_{0}^{+\infty} \frac{\mathrm{e}^{\mathrm{i} \tau} \mathrm{d} \tau}{\tau-\mathrm{i} z}=-\sum_{k=1}^{N}(k-1) ! z^{-k}+\frac{N !}{\mathrm{i}^{N}} \int_{0}^{\infty} \frac{\mathrm{e}^{\mathrm{i} \tau} \mathrm{d} \tau}{(\tau-\mathrm{i} z)^{N+1}} .
$$

Further we use the inequality $n^{-1}\left|\sum_{i=1}^{n} a_{i}\right| \leqslant\left(n^{-1} \sum_{i=1}^{n} a_{i}^{2}\right)^{1 / 2}$ (see 11.115 in Gradsteyn \& Ryzhik (1994)) to write

$$
|\tau-\mathrm{i} z|=\left[(\tau-\operatorname{Re}\{\mathrm{i} z\})^{2}+(\operatorname{Im}\{\mathrm{i} z\})^{2}\right]^{1 / 2} \geqslant 2^{-1 / 2}(\tau-\operatorname{Re}\{\mathrm{i} z\}+\operatorname{Im}\{\mathrm{i} z\})
$$

where we take into account signs of $\tau, \operatorname{Re}\{z\}$ and $\operatorname{Im}\{z\}$. Then we have

$$
\begin{aligned}
\left|\int_{0}^{\infty} \frac{\mathrm{e}^{\mathrm{i} \tau} \mathrm{d} \tau}{(\tau-\mathrm{i} z)^{N+1}}\right| & \leqslant \int_{0}^{\infty} \frac{\mathrm{d} \tau}{|\tau-\mathrm{i} z|^{N+1}} \leqslant 2^{(N+1) / 2} \int_{0}^{\infty} \frac{\mathrm{d} \tau}{(\tau+\operatorname{Re}\{z\}+\operatorname{Im}\{z\})^{N+1}} \\
& =2^{(N+1) / 2} N^{-1}(\operatorname{Re}\{z\}+\operatorname{Im}\{z\})^{-N} \leqslant 2^{(N+1) / 2} N^{-1}|z|^{-N} .
\end{aligned}
$$

The case $\operatorname{Im}\{z\}<0$ is analogous, in this case we use the formula

$$
\int_{0}^{\infty} \frac{\mathrm{e}^{-z t} \mathrm{~d} t}{t-1}-\pi \mathrm{ie}^{-z}=-\int_{-\infty}^{0} \frac{\mathrm{e}^{\mathrm{i} \tau} \mathrm{d} \tau}{\tau-\mathrm{i} z}
$$

Finally, combining (A.4) and (A.5) we find

where

$$
\operatorname{Ei}(z)=\pi \mathrm{i} \operatorname{sign}(\operatorname{Im}\{z\})+\mathrm{e}^{z}\left\{\sum_{n=1}^{N}(n-1) ! z^{-n}+R_{N}(z)\right\},
$$

$$
\begin{gathered}
R_{N}(z)=-\frac{N !}{\mathrm{i}^{N}} \int_{0}^{\infty} \frac{\mathrm{e}^{\mathrm{i} \tau} \mathrm{d} \tau}{(\tau-\mathrm{i} z)^{N+1}}, \\
\left|R_{N}\right| \leqslant 2^{(N+1) / 2}(N-1) !|z|^{-N}, \quad\left|R_{N}\right| \leqslant\left(2^{N / 2+1}+1\right) N !|z|^{-(N+1)} .
\end{gathered}
$$

Thus, by (A.3) and (A.6) with $N=1$ we arrive at

$$
\partial_{x} G-\mathrm{i} \partial_{y} G=-K \operatorname{sign}\left(x-x_{0}\right) \mathrm{e}^{K y} \mathrm{e}^{-\mathrm{i} K\left(x-x_{0}\right)}+\frac{\mathrm{i} K}{\pi} R_{1}(\mathrm{i} K \zeta),
$$

where the non-wave term is estimated as follows:

$$
\left|\frac{\mathrm{i} K}{\pi} R_{1}(\mathrm{i} K \zeta)\right| \leqslant R_{G}\left(\left|x-x_{0}\right|, y\right) \equiv \frac{1}{\pi} \min \left\{\frac{2}{r}, \frac{K(2 \sqrt{2}+1)}{(K r)^{2}}\right\} .
$$




\section{Appendix B. On derivatives of the three-dimensional Green function}

In the appendix we shall derive estimates for derivatives of the three-dimensional Green's function. By formula 6.611.1 from (Gradsteyn \& Ryzhik, 1994) and in view of the representation (2.3) we have for $Q \in F$

$$
G\left(\boldsymbol{x}, y ; \boldsymbol{x}_{0}, 0\right)=\frac{1}{2 \pi r}+\frac{K}{2 \pi} I(R, y), \quad I(R, y)=\int_{0}^{\infty} \frac{\mathrm{e}^{\mu y} \mathrm{~J}_{0}(\mu R)}{\mu-K} \mathrm{~d} \mu,
$$

where $r$ and $R$ are given in (2.2) and (5.4) respectively. By using formula 6.611.1 from (Gradsteyn \& Ryzhik, 1994) again, we find

$$
\partial_{y} I(R, y)=\frac{1}{r}+K I(R, y)
$$

The term $\mathrm{J}_{0}(\mu R)$ can be written as follows

$$
\mathrm{J}_{0}(\mu R)=\frac{1}{\pi} \int_{-1}^{1}\left(1-t^{2}\right)^{-1 / 2} \cos (\mu R t) \mathrm{d} t
$$

(see formula 8.411.8 in Gradsteyn \& Ryzhik (1994)). Substituting the latter representation to the definition of $I$ (B.1), changing order of integration and using the function Ei (cf. (A.2)) we get

$$
\begin{aligned}
I(R, y)= & \frac{1}{\pi} \int_{-1}^{1}\left(1-t^{2}\right)^{-1 / 2} \mathrm{~d} t \int_{0}^{\infty} \frac{\mathrm{e}^{\mu y} \cos (\mu R t)}{\mu-K} \mathrm{~d} \mu \\
= & -\frac{1}{\pi} \int_{-1}^{1}\left(1-t^{2}\right)^{-1 / 2} \operatorname{Re}\left\{\mathrm{e}^{-\mathrm{i} K \zeta(t)} \operatorname{Ei}(\mathrm{i} K \zeta(t))\right\} \mathrm{d} t \\
=-\frac{1}{\pi} \int_{-1}^{1}\left(1-t^{2}\right)^{-1 / 2} \operatorname{Re}\left\{\pi \mathrm{i} \operatorname{sign}(t) \mathrm{e}^{-\mathrm{i} K \zeta(t)}+\frac{1}{\mathrm{i} K \zeta(t)}\right. & \left.\quad-\frac{1}{K^{2}[\zeta(t)]^{2}}+R_{2}(\mathrm{i} K \zeta(t))\right\} \mathrm{d} t
\end{aligned}
$$

where $\zeta(t)=R t+\mathrm{i} y$ and the expansion (A.6) with $N=2$ is used. By formula 8.551.1 from (Gradsteyn \& Ryzhik, 1994) we have

$$
\begin{aligned}
\int_{-1}^{1}\left(1-t^{2}\right)^{-1 / 2} & \operatorname{Re}\left\{\pi \mathrm{i} \operatorname{sign}(t) \mathrm{e}^{-\mathrm{i} K \zeta(t)}\right\} \mathrm{d} t \\
= & 2 \pi \mathrm{e}^{K y} \int_{0}^{1}\left(1-t^{2}\right)^{-1 / 2} \sin (K R t) \mathrm{d} t=\pi^{2} \mathrm{e}^{K y} \mathbf{H}_{0}(K R),
\end{aligned}
$$

where $\mathbf{H}_{n}$ is the Struve function (Gradsteyn \& Ryzhik, 1994, sect. 8.55). We also find

$$
\int_{-1}^{1}\left(1-t^{2}\right)^{-1 / 2} \operatorname{Re}\left\{\frac{1}{\mathrm{i} K \zeta(t)}\right\} \mathrm{d} t=-\frac{y}{K} \int_{-1}^{1}\left(1-t^{2}\right)^{-1 / 2} \frac{\mathrm{d} t}{(R t)^{2}+y^{2}}=\frac{\pi}{K r},
$$




$$
\begin{array}{r}
\int_{-1}^{1}\left(1-t^{2}\right)^{-1 / 2} \operatorname{Re}\left\{\frac{1}{K^{2}[\zeta(t)]^{2}}\right\} \mathrm{d} t=\frac{1}{K^{2}} \int_{-1}^{1} \frac{\left(1-t^{2}\right)^{-1 / 2}\left(R^{2} t^{2}-y^{2}\right) \mathrm{d} t}{\left(R^{2} t^{2}-y^{2}\right)^{2}+4 R^{2} t^{2} y^{2}} \\
=\frac{\pi y}{K^{2} r^{3}}=-\frac{\pi}{K^{2}} \partial_{y}\left(r^{-1}\right)
\end{array}
$$

Thus, by (B.1)-(B.6) we arrive at

$$
\partial_{y} G\left(\boldsymbol{x}, y ; \boldsymbol{x}_{0}, 0\right)=-\frac{K^{2}}{2} \mathrm{e}^{K y} \mathbf{H}_{0}(K R)+\sigma_{1}(R, y)
$$

where

$$
\sigma_{1}(R, y)=-\frac{K^{2}}{2 \pi^{2}} \int_{-1}^{1}\left(1-t^{2}\right)^{-1 / 2} \operatorname{Re}\left\{R_{2}(\mathrm{i} K \zeta(t))\right\} \mathrm{d} t .
$$

Taking into account (A.7) and the equality $\int_{-1}^{1}\left(1-t^{2}\right)^{-1 / 2} \mathrm{~d} t=\pi$ we find

$$
\begin{aligned}
\left|\sigma_{1}(R, y)\right| & \leqslant \frac{K^{2}}{2 \pi^{2}} \max _{t \in[-1,1]}\left|R_{2}(\mathrm{i} K \zeta(t))\right| \int_{-1}^{1}\left(1-t^{2}\right)^{-1 / 2} \mathrm{~d} t \\
& \leqslant \frac{K^{2}}{2 \pi} \min \left\{\max _{t \in[-1,1]} \frac{2 \sqrt{2}}{|K \zeta(t)|^{2}}, \max _{t \in[-1,1]} \frac{10}{|K \zeta(t)|^{3}}\right\} \\
& =\frac{K^{2}}{2 \pi} \min \left\{\frac{2 \sqrt{2}}{K^{2} y^{2}}, \frac{10}{K^{3}|y|^{3}}\right\} .
\end{aligned}
$$

Consider now $\partial_{x} G$ and $\partial_{z} G$. We note that

$$
\partial_{x} G=\cos \alpha \partial_{R} G, \quad \partial_{z} G=\sin \alpha \partial_{R} G
$$

(see (5.4) for definition of $\alpha$ ) and, thus, $\partial_{R} G$ should only be treated. By using formulae 8.473.4 and 6.611.1 in (Gradsteyn \& Ryzhik, 1994), we find

$$
\partial_{R} I(R, y)=-\frac{r+y}{R r}-K I_{1}(R, y), \quad I_{1}(R, y)=\int_{0}^{\infty} \frac{\mathrm{e}^{\mu y} \mathrm{~J}_{1}(\mu R)}{\mu-K} \mathrm{~d} \mu .
$$

By using 8.411.8 from (Gradsteyn \& Ryzhik, 1994) and integrating by parts we write

$$
\begin{aligned}
I_{1}(R, y) & =\frac{R}{\pi} \int_{-1}^{1}\left(1-t^{2}\right)^{1 / 2} \mathrm{~d} t \int_{0}^{\infty} \frac{\mu \mathrm{e}^{\mu y} \cos (\mu R t)}{\mu-K} \mathrm{~d} \mu \\
& =\frac{1}{\pi} \int_{-1}^{1} t\left(1-t^{2}\right)^{-1 / 2} \mathrm{~d} t \int_{0}^{\infty} \frac{\mathrm{e}^{\mu y} \sin (\mu R t)}{\mu-K} \mathrm{~d} \mu .
\end{aligned}
$$

The principal value integral can be written in terms of exp and Ei (see (A.1)) and, then, in view of (A.6) we have

$$
\begin{aligned}
I_{1}(R, y)=\frac{1}{\pi} \int_{-1}^{1} t\left(1-t^{2}\right)^{-1 / 2} \operatorname{Im}\left\{\pi \mathrm{i} \operatorname{sign}(t) \mathrm{e}^{-\mathrm{i} K \zeta(t)}+\frac{1}{\mathrm{i} K \zeta(t)}\right. \\
\left.-\frac{1}{K^{2}[\zeta(t)]^{2}}+R_{2}(\mathrm{i} K \zeta(t))\right\} \mathrm{d} t .
\end{aligned}
$$


By 8.551.1 in (Gradsteyn \& Ryzhik, 1994),

$$
\begin{aligned}
& \int_{-1}^{1} t\left(1-t^{2}\right)^{-1 / 2} \operatorname{Im}\left\{\pi \mathrm{i} \operatorname{sign}(t) \mathrm{e}^{-\mathrm{i} K \zeta(t)}\right\} \mathrm{d} t \\
& \quad=2 \pi \mathrm{e}^{K y} \int_{0}^{1} t\left(1-t^{2}\right)^{-1 / 2} \cos (K R t) \mathrm{d} t=-\pi^{2} \mathrm{e}^{K y} \mathbf{H}_{1}(K R)+2 \pi \mathrm{e}^{K y} .
\end{aligned}
$$

Analogously to (B.5) and (B.6) we obtain

$$
\begin{aligned}
\int_{-1}^{1} t\left(1-t^{2}\right)^{-1 / 2} \operatorname{Im} & \left\{\frac{1}{\mathrm{i} K \zeta(t)}\right\} \mathrm{d} t \\
& =-\frac{R}{K} \int_{-1}^{1} t^{2}\left(1-t^{2}\right)^{-1 / 2} \frac{\mathrm{d} t}{(R t)^{2}+y^{2}}=-\frac{\pi(r+y)}{K R r}
\end{aligned}
$$

and

$$
\begin{aligned}
& \int_{-1}^{1} t\left(1-t^{2}\right)^{-1 / 2} \operatorname{Im}\left\{\frac{1}{K^{2}[\zeta(t)]^{2}}\right\} \mathrm{d} t \\
& \quad=-\frac{2 R y}{K^{2}} \int_{-1}^{1} \frac{t^{2}\left(1-t^{2}\right)^{-1 / 2} \mathrm{~d} t}{\left(R^{2} t^{2}-y^{2}\right)^{2}+4 R^{2} t^{2} y^{2}}=\frac{\pi R}{K^{2} r^{3}}=-\frac{\pi}{K^{2}} \partial_{R}\left(r^{-1}\right) .
\end{aligned}
$$

Finally, by (B.1) and (B.9)-(B.14) we arrive at

$$
\partial_{\left\{\begin{array}{l}
x \\
z
\end{array}\right\}} G\left(\boldsymbol{x}, y ; \boldsymbol{x}_{0}, 0\right)=\frac{K^{2}}{2 \pi}\left\{\begin{array}{c}
\cos \alpha \\
\sin \alpha
\end{array}\right\}\left[-\pi \mathrm{e}^{K y} \mathbf{H}_{1}(K R)+2 \mathrm{e}^{K y}+\sigma_{2}(R, y)\right],
$$

where

$$
\sigma_{2}(R, y)=\frac{1}{\pi} \int_{-1}^{1} t\left(1-t^{2}\right)^{-1 / 2} \operatorname{Im}\left\{R_{2}(\mathrm{i} K \zeta(t))\right\} \mathrm{d} t
$$

and in view of (A.7) and the equality $\int_{0}^{1} t\left(1-t^{2}\right)^{-1 / 2} \mathrm{~d} t=1$,

$$
\left|\sigma_{2}(R, y)\right| \leqslant \frac{K^{2}}{\pi^{2}} \min \left\{\frac{2 \sqrt{2}}{K^{2} y^{2}}, \frac{10}{|K y|^{3}}\right\}
$$

We summarize the results given in (B.7), (B.8), (B.15) and (B.16) and arrive at the sought estimate

$$
\left|\nabla G\left(\boldsymbol{x}, y ; \boldsymbol{x}_{0}, 0\right)\right| \leqslant K^{2}\left(\frac{1}{\pi^{2}}+\frac{1}{4}\right)^{1 / 2}\left[\mathrm{e}^{2 K y}+\pi^{-2} \min \left\{\frac{8}{(K y)^{4}}, \frac{100}{(K y)^{6}}\right\}\right]^{1 / 2} .
$$

Here we also used the inequalities $\left|\mathbf{H}_{0}(K R)\right| \leqslant 1,\left|\pi \mathbf{H}_{1}(K R)-2\right| \leqslant 2$, which obviously follows from (B.4) and (B.12). 